\title{
Analysis and Evaluation of Natural Diamond Cut by Water Jet - Guided Laser
}

\author{
Guangfeng $\mathrm{SHI}^{1} *$, Dongdong $\mathrm{HAN}^{2}$, Shukun $\mathrm{WANG}^{3}$, Keke ZHU \\ School of Mechanical and Electrical Engineering \\ Changchun University of Science and Technology \\ Changchun, China \\ e-mail: ${ }^{1}$ *shiguangfeng@ @ cust.edu.cn, ${ }^{2} 1354888500 @$ qq.com, ${ }^{3}$ wangshukun @ cust.edu.cn, ${ }^{4} 1198077882 @$ qq.com
}

\begin{abstract}
Natural diamond can be cut and grinded for manufacturing the super-hard tool in the field of ultraprecision machining. But the natural diamond cutting technology with high precision and high quality is still a challenge. The idea that laser cutting method combined with the other technologies provides a new try for natural diamond cutting. In this paper, water jet-guided laser (WJL) is used to cut the natural diamond along the crystal plane (111) for the diamond tool manufacture, and the cutting surfaces are observed by using optical microscope, optical profiler and $\mathrm{X}$ ray diffractometer to analyze the difference between the two cutting methods of laser and WJL. From the analyzed result of the morphology, roughness, phase composition and residential stress of the separated diamond surfaces, it is concluded that WJL is superior to the traditional laser cutting method, though its cutting process parameter should be further optimized for a better result.
\end{abstract}

\section{Keywords-natural diamond; WJL; laser; cut}

\section{INTRODUCTION}

Natural diamond has a very high hardness and it is colorful and durable, which can be made for expensive jewelry. Besides it is widely used in ultra-precision machining as a diamond tool and other industrial areas. But the high strength, hardness, and anisotropy of the natural diamond will bring great difficulties for manufacturing.

For natural diamond cutting, the traditional splitting method has some drawbacks of uncertainty and inaccuracy, and the surface after cutting is difficult to polish. While the saw cutting with a phosphor bronze blade will cost more material waste and time. To solve these problems, the noncontact cutting method using laser is more efficient to overcome the existing drawbacks, but it can't ignored that the heat affected zone, thermal stress and slag after cut will produce cracks and faults on the diamond cutting surfaces [1]. So some new cutting methods are essential for the promotion of natural diamond cutting application.

For a water jet-guided laser (WJL), the laser beam is guided through a hair-thin water jet, similar in principle to an optical fiber. WJL will drastically reduce damages with lower weight loss compared with dry lasers according to its machining principle. Total internal laser reflection by the stream of water will cause small heat effect on the post cut surface, and the water jet polish will get a better cutting surface at the meantime of inner laser function ${ }^{[2-4]}$.

At present, WJL has become a hot technology from experiment to application. But for the common users, it is difficult to master this high technology to get a satisfied result of natural diamond cutting, after all plenty of experiments for the process parameter is expensive. Maybe the new operator of the WJL machine think it is a good one but not easy to use, because there are many technological details of high-level analysis and understanding. Micromechanical behavior for crack or damage due to residual stresses and impact stress during the WJL machining is very important for the process parameters to be used to get a good result [5-6]. So in this paper, the morphology, roughness, phase composition and residential stress of the natural diamond cutting surfaces by using WJL are contrastively studied with the ones by using laser.

\section{WJL CUTTING PRINCIPLE AND SAMPLE}

Just like the company SYNOVA depicts for WJL: contained within a hair-thin water jet through total internal reflection, the laser beam surpasses today's laser and water cutting technologies. During machining, the work pieces are cooled by the water jet at the cutting interface, enabling cold laser cutting, with little or no thermal damage and negligible material changes, resulting in an exceptional high quality cut. At the same time, low water jet pressure ensures that virtually no mechanical force is exerted during processing, allowing rapid, damage free production of delicate and composite parts. The Laser achieves a precise cut over the entire depth of the work piece, leaving a fine, clean surface thanks to a long working distance and constant parallel laser beam. In the field of high-precision machining of sensitive materials, stringent requirements for fine and small structures demand a new process: water jet-guided laser is the solution. So from the above, we can know the WJL cutting principle, and the WJL cutting process is shown in Fig.1.
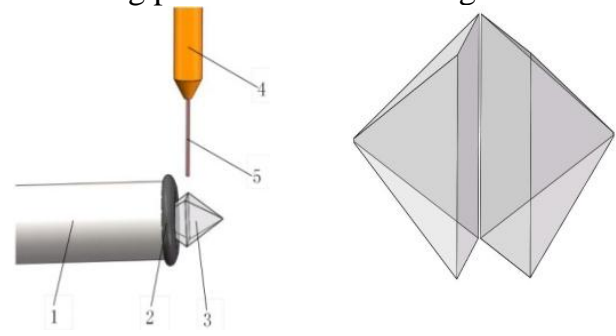

Figure 1. WJL cutting process:1.fixture, 2. adhesive, 3. natural diamond, 4.WJL spray head, 5. Nozzle.

With the WJL machine produced by SYNOVA, several natural diamond samples were cut along the crystal plane (111) by our fellows, as shown in Fig.2. After transferred by different people, we can't know the exact machining process. 
But with many advanced test equipments, the observed result can be shown step by step.

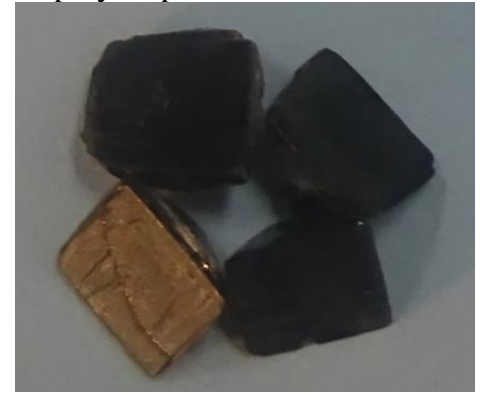

Figure 2. Samples cut by WJL and laser.

\section{TEST RESULT ANALYSIS}

\section{A. Surface Morphology}

For observing with optical microscope, the diamond surfaces washed with alcohol are shown in Fig.3. The diamond surface cut by laser is often covered with a black reactant wrapped, which is ablated with cracks and faults by the high temperature [3]. There are also burrs and other defects along the diamond edge as shown in Fig.4 (a). While the surface cut by WJL is semi transparent with the cutting texture locally, though there may be a change of cutting direction for the whole cut.

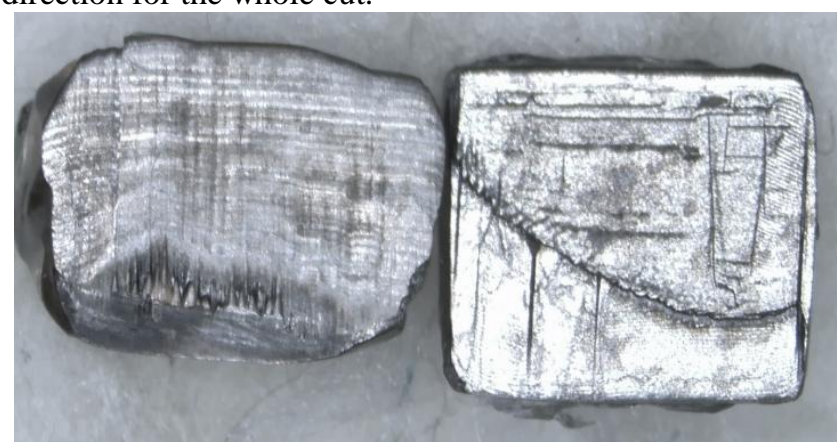

Figure 3. Laser cutting (left) and WJL cutting (right).

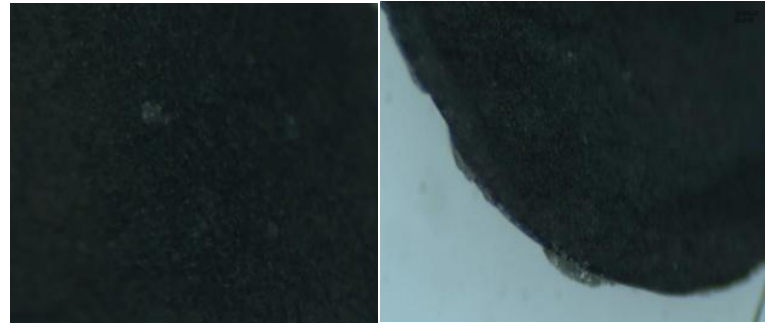

(a) Laser cutting surface (left) and its edge burr (right)

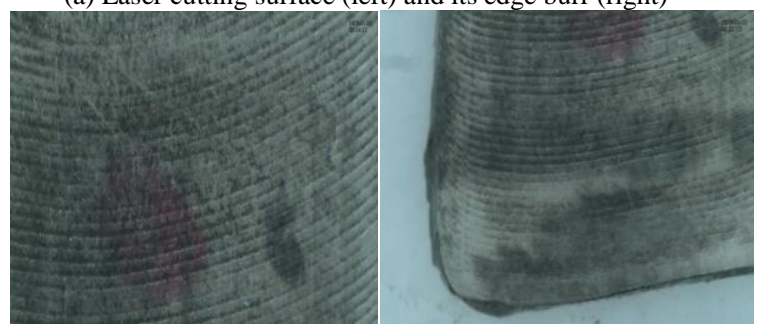

(b) WJL cutting surface (left) and its edge burr (right)

Figure 4. Different cutting results under magnifier.
Our fellows have told us that a necessary change of cutting direction did happen because a smaller laser power of water jet pressure were used. So it was difficult to cut the natural diamond off at once, but changing a cutting direction from the opposite side for small resistance is a good idea. The demarcation line may be a precision problem of the revolving fixture or some other reasons. Of course, a holonomic cutting surface can be got when a higher energy of the WJL is used.

The cutting tracks on the diamond surface of WJL cutting (Fig.4 (b)) indicate that the cutting jet at the end of the diamond material met a lot of resistance so that the straight cutting direction changed and moving back wholly.

\section{B. Surface Roughness}

In the following, two kinds of the surfaces are tested by using Wyko NT1100 optical profiler. In order to ensure the reliability of the test data, the method of multi-point measurement is used for analysis. As shown in Fig.5 and Fig.6, the measurement results show that the Ra value of WJL cutting surface is about $400 \mathrm{~nm}$, which is nearly twice of the Ra value $200 \mathrm{~nm}$ of laser cutting surface. Obviously, the WJL cutting tracks greatly affects the roughness.

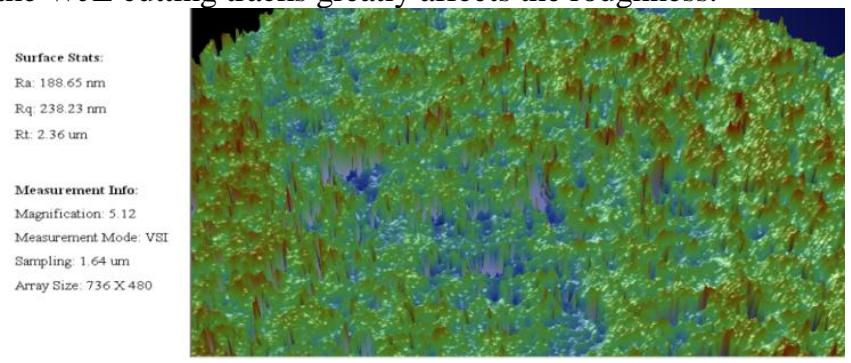

Figure 5. Roughness of Laser cutting surface.

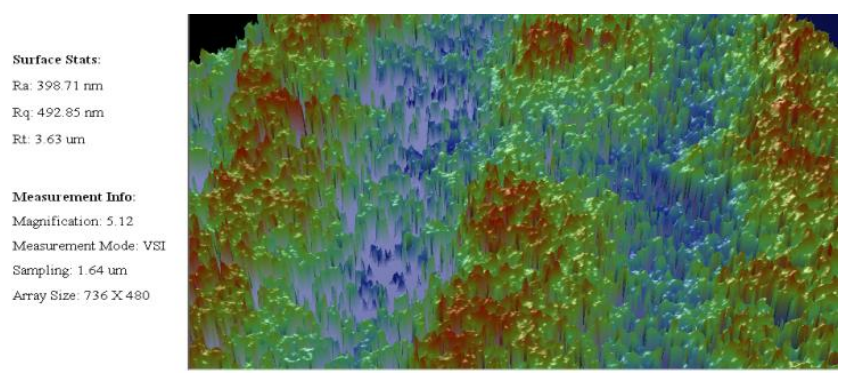

Figure 6. Roughness of WJL cutting surface.

It seems contradictory, but it indicates that there is an optimization for the process parameters. Because the ablated layer of the laser cutting surface and the higher roughness of the WJL cutting surface both need a large thickness of removal rate for post polishing. If the process parameters can't be set reasonably, there will be no desired result of WJL cutting.

But the ablation effect is unavoidable for laser cutting, which uses a conventional optical system with the problem of precise focusing. And the effect of the defocusing angle on the coupling condition of laser will result in the ablation phenomenon [4]. While the WJL can solve this problem and 
its cutting tracks can be eliminated by reducing the amount of feed though the machining time will increase.

\section{Phase Composition}

The above analysis is about the external morphology representation, while the material characteristics will be another interesting topics. So in the following, the Panalytical Empyrean X-ray diffractometer (XRD) is used for the phase composition analysis of the cutting surfaces, as shown in Fig.7. The sample with the cutting surface upward is attached to the top center of the rotation stable.

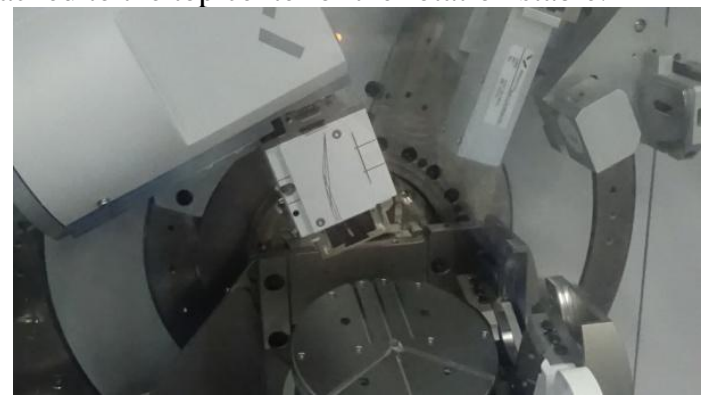

Figure 7. XRD test of the diamond cutting surface.

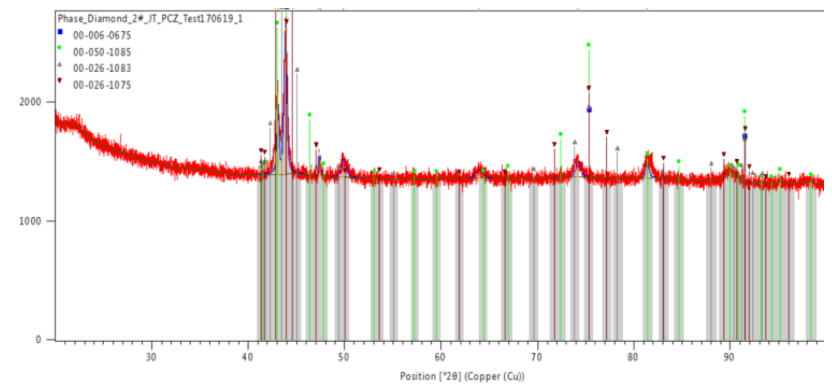

Figure 8. Diamond deep phase.

The phase results of the natural diamond tested by XRD are shown in Fig. 8-Fig.10. The results of the deep phase test show that the strongest peaks of the samples are diamond diffraction peak, which indicates that the interior material is mainly diamond component, as shown in Fig.8.

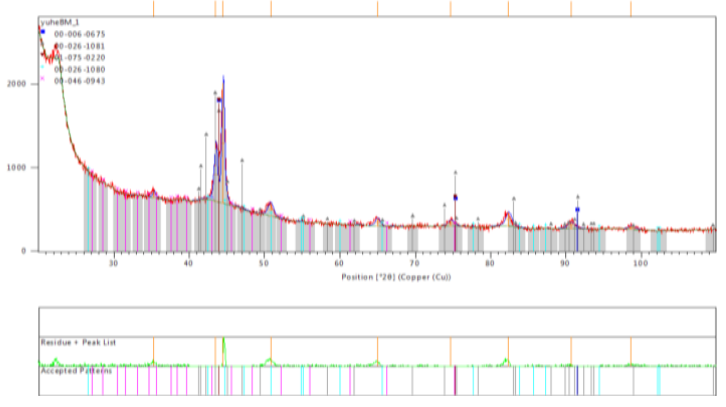

Figure 9. WJL cutting surface phase.

And the other small peaks of WJL cutting surface indicate that the diamond material contains a great amount of trace impurities but not graphite, as in Fig.9. While the small peak is carbon allotrope, which indicates that there are some changes from diamond to carbon allotrope. But in the laser cutting surface, the fifth diffraction peak is graphite with the rest being also carbon allotrope, as shown in Fig.10. So the results prove the cooling effect of WJL cutting method.
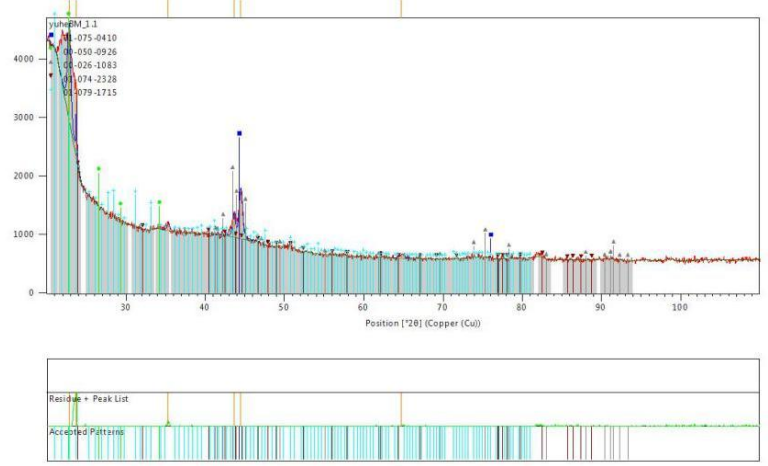

Figure 10. Laser cutting surface phase.

TABLE I. PARAMETERS COMPARISON

\begin{tabular}{|c|c|c|c|}
\hline $\boldsymbol{\Psi}$ & $\sin \mathbf{2} \boldsymbol{}$ & $\mathbf{d}(\mathbf{A}) \mathbf{W J L}$ & d(A)Laser \\
\hline $0^{\circ}$ & 0 & 1.1746 & 1.1748 \\
\hline $18.43^{\circ}$ & 0.1 & 1.1743 & 1.1741 \\
\hline $26.57^{\circ}$ & 0.2 & 1.1734 & 1.1734 \\
\hline $33.21^{\circ}$ & 0.3 & 1.1728 & 1.1726 \\
\hline $39.23^{\circ}$ & 0.4 & 1.1725 & 1.1725 \\
\hline $45^{\circ}$ & 0.5 & 1.1719 & 1.1718 \\
\hline
\end{tabular}

The data in Table I. are the crystal lattice parameters obtained by the relative computation. It can be seen that the crystal lattice parameters of the two kinds of diamond cutting surfaces are similar, which will produce similar residual stress (tensile stress) with each other [5-6]. So this phenomenon can't show the advantage of WJL over laser.

The tensile stress is reflected by the crystal lattice parameters change, which will become compression stress after the cutting surfaces are polished no matter what kind of cutting because compression stress has been tested on several polished surfaces after the two kinds of cutting. So if the polishing pressure is over forced, the residual stress caused by the cutting will be released and result in micro cracks on the cutting surface even invalid for using. But the cutting methods will affect little on the residual stress, and the micro cracks on the cutting surface may be mainly due to the post polishing process.

\section{CONCLUSIONS}

The ablated layer of the laser cutting surface and the higher roughness of the WJL cutting surface both need a large thickness of removal rate for post polishing when the process parameters are not chosen rightly, and it needs an optimization for a set of better process parameters to get an ideal cutting result. The high temperature of natural diamond surfaces yielded by laser cutting will make natural diamond change into graphite. While the natural diamond surface cut by WJL only forms a thin layer of carbon allotrope covered on the diamond surface. The cutting methods will affect little on the residual stress, and the micro cracks on the cutting surface may be mainly due to the post polishing process. WJL can be adjusted to achieve the high efficiency and high quality of diamond cutting. 


\section{ACKNOWLEDGMENT}

This work was financially supported by the Jilin province science and technology development projects (No. 20150204 059GX, No. 20170101124JC), and another subordinate project named international science and technology cooperation project which is just approved but without the approved number. Further more, this work was also surported by National Natural Science Foundation of China (No.51405031,No.51575075).

\section{REFERENCES}

[1] Gamaly E G, Rode A V, Uteza O, et al. Control over a phase state of the laser plume ablated by femtosecond laser: Spatial pulse shaping[J]. Journal of Applied Physics, 2004, 104(5): 2250-2257.
[2] Frank Wagne, Ochelio Sibailly, Nandor Vago, Rafal Romanowicz,Bermold Richerzhagen Svnova SA Fcublens CH The Laser Micoiet Technology-10 Years of Development (M401) [J]. 2003:1-9.

[3] Bernold Richerzhagen. Induserial Applications of the Water-jet Guided Laser[J].Laser in Manufacturing.2002, 28:28-30.

[4] Daniel Kraya, Sybille Hopmana, Akos Spiegel, et al. Study on the edge isolation of industrial silicon solar cells with waterjet-guided laser[J].Solar Energy Materials and Solar Cells,2007,91(17):16381644

[5] WITHERS P J. Residual Stress Part 1: Measurement Techniques[J]. Materials Science and Technology,2001,17:355-365.

[6] WITHERS P J. Residual Stress Part 2: Nature and Origins [J]. Materials Science and Technology, 2001, 17:366-375. 